\title{
Stable Sarma State in Two-band Fermi Systems
}

\author{
Lianyi He and Pengfei Zhuang \\ Physics Department, Tsinghua University, Beijing 100084, China
}

\begin{abstract}
We investigate fermionic superconductivity with mismatched Fermi surfaces in a general twoband system. The exchange interaction between the two bands changes significantly the stability structure of the pairing states. The Sarma state with two gapless Fermi surfaces which is always unstable in single-band systems, can be the stable ground state in two-band systems. To realize a visible mismatch window for the stable Sarma state, two conditions should be satisfied: a nonzero inter-band exchange interaction and a large asymmetry between the two bands.
\end{abstract}

PACS numbers: 74.20.-z, 03.75.Kk, 05.30.Fk

\section{INTRODUCTION}

The Cooper pairing with mismatched Fermi surfaces, which has been investigated many years ago $\underline{1.2}$, promoted new interest in the study of new superconducting materials in strong magnetic field and ultracold fermions due to the realization of superfluidity in resonantly interacting Fermi gases. The well-known theoretical result for s-wave weak coupling superconductors is that, at a critical mismatch, called Chandrasekhar-Clogston limit (CC limit) $h_{c}=0.707 \Delta_{0}$ where $\Delta_{0}$ is the zero temperature gap, a first order phase transition from the gapped BCS state to the normal state occurs ${ }^{3}$. Further theoretical studies showed that the inhomogeneous Fulde-Ferrell-LarkinOvchinnikov (FFLO) state ${ }^{2}$ may survive in a narrow window between $h_{c}$ and $h_{\mathrm{FFLO}}=0.754 \Delta_{0}$. However, since the thermodynamic critical field is much smaller than the $\mathrm{CC}$ limit due to strong orbit effect $\mathrm{3}^{3}$, it is hard to observe the CC limit and the FFLO state in ordinary superconductors. In recent years, some experimental evidence for the FFLO state in heavy fermion superconductors ${ }^{4}$, high temperature supercondutors ${ }^{\frac{5}{5}}$ and organic superconductors ${ }^{6}-$ were found. More recently, in the study of ultracold atoms, Fermi superfluidity with population imbalance was realized by MIT and Rice groups independently ${ }^{7}$. The ultracold fermion experiments has promoted a lot of theoretical works $8.9,10,11$ on the superfluidity mechanism and the phase diagrams for the crossover from Bardeen-Cooper-Schrieffer (BCS) to Bose-Einstein Condensation(BEC) $\stackrel{12}{\underline{12}}$. The problem of imbalanced pairing is also related to the study of color superconductivity and pion superfluidity in dense quark matter ${ }^{13,14}$.

While most of the theoretical works focus on the inhomogeneous FFLO state, we in this paper are interested in the homogeneous and gapless Sarma state ${ }^{1}$. For weak coupling superconductors, the Sarma state is located at the maximum of the thermodynamic potential of the system, and therefore can not be the stable ground state. This was called Sarma instability many years ago $\underline{1}$. The thermodynamic instability of the Sarma state can be traced to the existence of gapless fermion excitations which cause a very large density of state at the gapless Fermi surfaces 1,2 . To realize a stable Sarma state, one should have some mechanism to cure the instabil- ity. Forbes et.al $\stackrel{16}{\underline{n}}$ proposed that, a stable Sarma state is possible in a model with finite range interaction where the momentum dependence of the pairing gap cures the instability. On the other hand, when the attractive interaction becomes strong enough which can be realized in ultracold fermion experiments, the stability of the Sarma state can be changed. While the homogeneous Sarma state is always unstable at the BCS side of the BCSBEC crossover, it becomes stable in the deep BEC region ${ }^{9}$. However, this stable Sarma state at the BEC side is not the original "interior gap" or "breached pairing" state with two gapless Fermi surfaces proposed by Liu and Wilczek ${ }^{15}$. Since the fermion chemical potential becomes negative in the BEC region, the Sarma state in this case possesses only one gapless Fermi surface, and the matter behaves like a Bose-Fermi mixture $\underline{17}$.

In this paper, we focus on how the multi-band structure which may be realized in solid materials and optical lattices changes the stability of the Sarma state. We consider a general two-band Fermi system, and show that the inter-band exchange interaction can cure the Sarma instability and the Sarma state can be the stable ground state in visible parameter regions.

The multi-band theory of BCS superconductivity was firstly introduced by Suhl et al $\stackrel{18}{i n} 1959$ to describe the possible multiple band crossings at the Fermi surface. The two-band model has been applied to the study of high- $T_{c}$ superconductors 19 to effectively describe the particular crystalline and electronic structure. Recently, it is found that, the material $\mathrm{MgB}_{2}$ is a standard two-band superconductor ${ }^{20}$ and many experimental data can be explained by the two-band model of BCS superconductivity. Multi-band Fermi systems may be realized experimentally with ultracold atoms in optical lattice $\stackrel{21}{\underline{1}}$. For example, if we confine the cold atoms in a one dimensional periodic external potential, the band structure will form in the confined direction, and the matter can be regarded as a multi-band system in two-dimensions. In this case, by adjusting the coupling strength, one can study the possible BCS-BEC crossover in multi-band systems 19,22 . The inter-band physics in optical lattices is recently studied $^{23}$, and the multi-gap superfluidity is also possible in nuclear matter 24 .

The paper is organized as follows. In Section II we 
give an introduction to the Sarma state in single-band systems. We discuss the stability of the Sarma state in a general two-band model in Section III and summarize in Section IV.

\section{SARMA STATE IN SINGLE-BAND MODEL}

Before discussing the stability of Sarma state in twoband systems, we in this section give a brief introduction to the Sarma state in single-band systems. We start from the following standard BCS-type Hamiltonian

$$
\begin{aligned}
H & =\int d^{3} \mathbf{r}\left[\sum_{\sigma} \psi_{\sigma}^{\dagger}(\mathbf{r})\left(-\frac{\nabla^{2}}{2 m}-\mu_{\sigma}\right) \psi_{\sigma}(\mathbf{r})\right. \\
& \left.-U \psi_{\uparrow}^{\dagger}(\mathbf{r}) \psi_{\downarrow}^{\dagger}(\mathbf{r}) \psi_{\downarrow}(\mathbf{r}) \psi_{\uparrow}(\mathbf{r})\right] .
\end{aligned}
$$

We constrain ourselves to discuss systems at zero temperature where the BCS mean field theory can be applied even at strong coupling ${ }^{12}$. In the mean field approximation, the Hamiltonian is approximated by

$$
\begin{aligned}
H_{\mathrm{mf}} & =\int d^{3} \mathbf{r}\left[\sum_{\sigma} \psi_{\sigma}^{\dagger}(\mathbf{r})\left(-\frac{\nabla^{2}}{2 m}-\mu_{\sigma}\right) \psi_{\sigma}(\mathbf{r})\right. \\
& \left.+\Phi(\mathbf{r}) \psi_{\uparrow}^{\dagger}(\mathbf{r}) \psi_{\downarrow}^{\dagger}(\mathbf{r})+\text { H.c. }+\frac{|\Phi(\mathbf{r})|^{2}}{U}\right],
\end{aligned}
$$

where $\Phi(\mathbf{r})=-U\left\langle\psi_{\downarrow}(\mathbf{r}) \psi_{\uparrow}(\mathbf{r})\right\rangle$ is the order parameter field of superconductivity. For homogeneous superconductivity, the thermodynamic potential $\Omega$ can be obtained by using the standard diagonal method ${ }^{18}$. It can be expressed as

$$
\Omega=\frac{\Delta^{2}}{U}+\int \frac{d^{3} \mathbf{k}}{(2 \pi)^{3}}\left[\left(\xi_{\mathbf{k}}-E_{\mathbf{k}}\right)+\sum_{\sigma} E_{\mathbf{k}}^{\sigma} \Theta\left(-E_{\mathbf{k}}^{\sigma}\right)\right]
$$

with the definition of energy dispersions $\xi_{\mathbf{k}}=\mathbf{k}^{2} /(2 m)-$ $\mu, E_{\mathbf{k}}=\sqrt{\xi_{\mathbf{k}}^{2}+\Delta^{2}}, E_{\mathbf{k}}^{\uparrow}=E_{\mathbf{k}}+h$ and $E_{\mathbf{k}}^{\downarrow}=E_{\mathbf{k}}-h$, where $\mu=\left(\mu_{\uparrow}+\mu_{\downarrow}\right) / 2$ and $h=\left(\mu_{\uparrow}-\mu_{\downarrow}\right) / 2$ are, respectively, the averaged and mismatched chemical potentials, and $\Delta$ is the modulus of $\Phi(\mathbf{r})$.

Without loss of generality, we set $h \geq 0$. The possible ground state of the system corresponds to the stationary point of the thermodynamic potential $\Omega$. This gives the so-called gap equation

$$
\left(\frac{1}{U}-\int \frac{d^{3} \mathbf{k}}{(2 \pi)^{3}} \frac{\Theta\left(E_{\mathbf{k}}^{\downarrow}\right)}{2 E_{\mathbf{k}}}\right) \Delta=0 .
$$

To properly achieve strong coupling, the chemical potentials should be renormalized by the number equations. The number density $n$ and spin density imbalance $\delta$ can be evaluated as

$$
\begin{aligned}
n & =n_{\uparrow}+n_{\downarrow}=\int \frac{d^{3} \mathbf{k}}{(2 \pi)^{3}}\left[1-\frac{\xi_{\mathbf{k}}}{E_{\mathbf{k}}} \Theta\left(E_{\mathbf{k}}^{\downarrow}\right)\right], \\
\delta & =n_{\uparrow}-n_{\downarrow}=\int \frac{d^{3} \mathbf{k}}{(2 \pi)^{3}} \Theta\left(-E_{\mathbf{k}}^{\downarrow}\right) .
\end{aligned}
$$

Whether the Zeeman energy imbalance $h$ or the spin density imbalance $\delta$ is experimentally adjusted depends on detailed systems. For cold atoms, the spin density imbalance $\delta$ is directly tuned, but in superconductors, the Zeeman splitting $h$ is adjusted via an external magnetic field.

\section{A. Stability Analysis}

If a solution of the gap equation is the ground state of the system, it should be the global minimum of the thermodynamic potential $\Omega^{16,26}$. The condition for a local minimum of $\Omega$ is that

$$
\frac{\partial \Omega(\Delta)}{\partial \Delta}=0, \quad \frac{\partial^{2} \Omega(\Delta)}{\partial \Delta^{2}}>0 .
$$

The first condition corresponds to the gap equation and the second order derivative $I=\partial^{2} \Omega(\Delta) / \partial \Delta^{2}$ can be evaluated as

$$
I=\int \frac{d^{3} \mathbf{k}}{(2 \pi)^{3}} \frac{\Delta^{2}}{E_{\mathbf{k}}^{2}}\left[\frac{\Theta\left(E_{\mathbf{k}}^{\downarrow}\right)}{E_{\mathbf{k}}}-\delta\left(E_{\mathbf{k}}^{\downarrow}\right)\right] .
$$

Let us study the Sarma state with $h>\Delta$ which induces a nonzero spin density imbalance $\delta$. At weak coupling, $I$ can be approximately evaluated as

$$
\frac{\pi^{2} I}{m} \simeq \sqrt{2 m \mu}\left[1-\frac{h \Theta(h-\Delta)}{\sqrt{h^{2}-\Delta^{2}}}\right]
$$

which shows that $\partial^{2} \Omega(\Delta) / \partial \Delta^{2}$ is always negative and therefore the Sarma state is unstable.

To achieve the BCS-BEC crossover, we renormalize the coupling constant with the two-body scattering length $a_{s}$,

$$
\frac{m}{4 \pi a_{s}}=-\frac{1}{U}+\int \frac{d^{3} \mathbf{k}}{(2 \pi)^{3}} \frac{m}{\mathbf{k}^{2}}
$$

In this case, we first solve the coupled gap and number equations at fixed total density $n=k_{\mathrm{F}}^{3} /\left(3 \pi^{2}\right)$. The result can be expressed ${ }^{9}$ as a function of the dimensionless coupling parameter $g=1 /\left(k_{\mathrm{F}} a_{s}\right)$ and the population imbalance $P=\delta / n$. The numerical calculations show that, the key quantity $I$ is always negative at the BCS side of the resonance $\left(a_{s}<0, g<0\right)$ where the Sarma state has two gapless Fermi surfaces, but the Sarma state can be a stable ground state in the strong coupling BEC region (roughly for $g>2.2$ ) where the chemical potential $\mu$ become negative. However, this Sarma state has only one gapless Fermi surface and is different from the Fermi surface topology of the so-called breached pairing state.

\section{B. Solution at Weak Coupling}

At weak coupling where the chemical potential $\mu$ is well approximated by the Fermi energy $\epsilon_{\mathrm{F}}$, the gap equation 
can be approximated by

$$
\left[\frac{1}{U N}-\int_{0}^{\Lambda} d \xi \frac{\Theta\left(\sqrt{\xi^{2}+\Delta^{2}}-h\right)}{\sqrt{\xi^{2}+\Delta^{2}}}\right] \Delta=0
$$

where $N$ is the density of state for each spin state at the Fermi surface, and $\Lambda$ is the energy cutoff which plays the role of Debye energy $\hbar \omega_{\mathrm{D}}$ in solids. After the integration and using the condition $\Delta \ll \Lambda$, we find

$$
\left[\frac{1}{U N}-\ln \frac{2 \Lambda}{\Delta}+\Theta(h-\Delta) \ln \frac{h+\sqrt{h^{2}-\Delta^{2}}}{\Delta}\right] \Delta=0
$$

There are three possible solutions to the gap equation (11) for $h \neq 0$. The first is the trivial normal phase with $\Delta_{\mathrm{N}}=0$. The second corresponds to the ordinary fully gapped BCS solution satisfying $\Delta>h$,

$$
\Delta_{\mathrm{BCS}}=\Delta_{0}=2 \Lambda e^{-1 /(U N)} .
$$

The third solution, i.e., the gapless Sarma state satisfying $\Delta<h$, can be analytically evaluated via the comparing with the BCS solution. It is 25

$$
\Delta_{\mathrm{S}}=\sqrt{\Delta_{0}\left(2 h-\Delta_{0}\right)}
$$

Using the weak coupling approximation, the grand potential $\Omega$ for various solutions can be expressed as

$$
\begin{aligned}
\Omega & =\frac{\Delta^{2}}{U}+2 N \int_{0}^{\Lambda} d \xi\left[\xi-\sqrt{\xi^{2}+\Delta^{2}}\right. \\
& \left.+\left(\sqrt{\xi^{2}+\Delta^{2}}-h\right) \Theta\left(h-\sqrt{\xi^{2}+\Delta^{2}}\right)\right] .
\end{aligned}
$$

Performing the integral over $\xi$, and using the condition $\Delta \ll \Lambda$ as well as the gap equation to cancel the cutoff dependence, we have

$$
\Omega=-\frac{N}{2} \Delta^{2}-\Theta(h-\Delta) N h \sqrt{h^{2}-\Delta^{2}} .
$$

Note that we have set the grand potential of the normal state at $h=0$ to be zero, $\Omega_{\mathrm{N}}(h=0)=0$. To see why the Sarma state is always thermodynamically unstable, one should calculate the grand potential differences between Sarma and other two states ${ }^{25}$,

$$
\begin{aligned}
\Omega_{\mathrm{S}}-\Omega_{\mathrm{BCS}} & =N\left(\Delta_{0}-h\right)^{2}, \\
\Omega_{\mathrm{S}}-\Omega_{\mathrm{N}} & =\frac{N}{2}\left(\Delta_{0}-2 h\right)^{2},
\end{aligned}
$$

which confirm that the Sarma state always has higher potential than the BCS and normal states. As a consequence, there exists a first order phase transition from BCS state to normal state. From the result

$$
\Omega_{\mathrm{BCS}}-\Omega_{\mathrm{N}}=\frac{N}{2}\left(2 h^{2}-\Delta_{0}^{2}\right),
$$

the transition occurs at the CC limit of BCS superconductivity, $h_{c}=\Delta_{0} / \sqrt{2}$.

\section{SARMA STATE IN TWO-BAND MODEL}

We in this section turn to the two-band model. Since the goal of this paper is to search for the possibility of stable Sarma state in general two-band Fermi systems, we consider a continuum Hamiltonian and neglect the details of the band structure in different systems. We will show that the key point is the inter-band scattering which can make the Sarma state stable in multi-band systems. The possible complicated lattice structure in various materials and optical lattices will not qualitatively change our conclusion. The obtained conclusion is generic and may be useful for the study of superconducting materials and ultracold atom gases.

The continuum Hamiltonian of the two-band model can be written as 18

$$
\begin{aligned}
H & =\int d^{3} \mathbf{r}\left[\sum_{\nu, \sigma} \psi_{\nu \sigma}^{\dagger}(\mathbf{r})\left(-\frac{\nabla^{2}}{2 m_{\nu}}-\mu_{\nu \sigma}\right) \psi_{\nu \sigma}(\mathbf{r})\right. \\
& \left.-\sum_{\nu, \lambda} U_{\nu \lambda} \psi_{\nu \uparrow}^{\dagger}(\mathbf{r}) \psi_{\nu \downarrow}^{\dagger}(\mathbf{r}) \psi_{\lambda \downarrow}(\mathbf{r}) \psi_{\lambda \uparrow}(\mathbf{r})\right]
\end{aligned}
$$

where $\nu, \lambda=1,2$ denote the band and $\sigma=\uparrow, \downarrow$ the direction of fermion spin. In superconductors, the band degrees of freedom usually come from the particular crystalline and electronic structure of the materials. In ultracold atom gases, these degrees of freedom may come from different hyperfine states or different atom species or the external periodic lattice potential. In general case, the effective fermion mass depends only on the band index, but the chemical potential is related to both the band and spin indexes due to the existence of external magnetic field or population imbalance. The constants $U_{11} \equiv U_{1}$ and $U_{22} \equiv U_{2}$ are the intra-band couplings, and $U_{12}=U_{21} \equiv J$ is the inter-band exchange coupling. For vanishing $J$, the model is reduced to a simple system with two independent bands. In the following we focus on how the inter-band coupling $J$ changes the stability of the Sarma state.

We first calculate the thermodynamic potential of the two-band Hamiltonian. In the mean field approximation, the Hamiltonian is approximated by

$$
\begin{aligned}
H_{\mathrm{mf}} & =\int d^{3} \mathbf{r}\left\{\sum_{\nu, \sigma} \psi_{\nu \sigma}^{\dagger}(\mathbf{r})\left(-\frac{\nabla^{2}}{2 m_{\nu}}-\mu_{\nu \sigma}\right) \psi_{\nu \sigma}(\mathbf{r})\right. \\
& +\sum_{\nu}\left[\Phi_{\nu}(\mathbf{r}) \psi_{\nu \uparrow}^{\dagger}(\mathbf{r}) \psi_{\nu \downarrow}^{\dagger}(\mathbf{r})+\text { H.c. }\right] \\
& +\frac{1}{G}\left[U_{2}\left|\Phi_{1}(\mathbf{r})\right|^{2}+U_{1}\left|\Phi_{2}(\mathbf{r})\right|^{2}\right. \\
& \left.\left.-J\left(\Phi_{1}^{*}(\mathbf{r}) \Phi_{2}(\mathbf{r})+\Phi_{2}^{*}(\mathbf{r}) \Phi_{1}(\mathbf{r})\right)\right]\right\}
\end{aligned}
$$

where $\Phi_{\nu}(\mathbf{r})=-\sum_{\lambda} U_{\nu \lambda}\left\langle\psi_{\lambda \downarrow}(\mathbf{r}) \psi_{\lambda \uparrow}(\mathbf{r})\right\rangle$ are two order parameter fields of the superconductivity, and $G$ is defined as $G=U_{1} U_{2}-J^{2}$. For homogeneous superconductivity, the thermodynamic potential $\Omega$ of this two-band 
model can be obtained by using the standard diagonal $\operatorname{method}^{18}$. It can be expressed as

$$
\begin{aligned}
\Omega & =\frac{1}{G}\left[U_{2} \Delta_{1}^{2}+U_{1} \Delta_{2}^{2}-2 J \Delta_{1} \Delta_{2} \cos \left(\varphi_{1}-\varphi_{2}\right)\right] \\
& +\sum_{\nu} \int \frac{d^{3} \mathbf{k}}{(2 \pi)^{3}}\left[\left(\xi_{\mathbf{k} \nu}-E_{\mathbf{k} \nu}\right)+\sum_{\sigma} E_{\mathbf{k} \nu}^{\sigma} \Theta\left(-E_{\mathbf{k} \nu}^{\sigma}\right)\right]
\end{aligned}
$$

with the definition of energy dispersions $\xi_{\mathbf{k} \nu}=$ $\mathbf{k}^{2} /\left(2 m_{\nu}\right)-\mu_{\nu}, E_{\mathbf{k} \nu}=\sqrt{\xi_{\mathbf{k} \nu}^{2}+\Delta_{\nu}^{2}}, E_{\mathbf{k} \nu}^{\uparrow}=E_{\mathbf{k} \nu}+h_{\nu}$ and $E_{\mathbf{k} \nu}^{\downarrow}=E_{\mathbf{k} \nu}-h_{\nu}$, where $\mu_{\nu}=\left(\mu_{\nu \uparrow}+\mu_{\nu \downarrow}\right) / 2$ and $h_{\nu}=\left(\mu_{\nu \uparrow}-\mu_{\nu \downarrow}\right) / 2$ are, respectively, the averaged and mismatched chemical potentials, and $\Delta_{\nu}$ the modulus of $\Phi_{\nu}$ and $\varphi_{\nu}$ their phases through the definition $\Phi_{\nu}=$ $\Delta_{\nu} e^{i \varphi_{\nu}}$. Without loss of generality, we take $h_{\nu}>0$. For $J>0$, the choice of $\varphi_{1}=\varphi_{2}$ is favored, otherwise there is $\varphi_{1}=\varphi_{2}+\pi$. We assume $J>0$ and set $\varphi_{1}=\varphi_{2}$.

The possible ground state of the system corresponds to the stationary point of the thermodynamic potential $\Omega$. This gives the so-called gap equations

$$
\left(\frac{U_{\bar{\nu} \bar{\nu}}}{G}-\int \frac{d^{3} \mathbf{k}}{(2 \pi)^{3}} \frac{\Theta\left(E_{\mathbf{k} \nu}^{\downarrow}\right)}{2 E_{\mathbf{k} \nu}}\right) \Delta_{\nu}-\frac{J}{G} \Delta_{\bar{\nu}}=0
$$

with $\bar{\nu}=1$ for $\nu=2$ and $\bar{\nu}=2$ for $\nu=1$. The gap equations are essentially the same as derived in $\underline{18}$. To properly achieve strong coupling, the chemical potentials should be renormalized by the number equations. The number equations for the fermion density $n_{\nu}$ and density imbalance $\delta_{\nu}$ for the $\nu$-th band can be evaluated as

$$
\begin{aligned}
& n_{\nu}=n_{\nu \uparrow}+n_{\nu \downarrow}=\int \frac{d^{3} \mathbf{k}}{(2 \pi)^{3}}\left[1-\frac{\xi_{\mathbf{k} \nu}}{E_{\mathbf{k} \nu}} \Theta\left(E_{\mathbf{k} \nu}^{\downarrow}\right)\right], \\
& \delta_{\nu}=n_{\nu \uparrow}-n_{\nu \downarrow}=\int \frac{d^{3} \mathbf{k}}{(2 \pi)^{3}} \Theta\left(-E_{\mathbf{k} \nu}^{\downarrow}\right),
\end{aligned}
$$

and the total density $n$ and total density imbalance $\delta$ of the system are defined as $n=n_{1}+n_{2}$ and $\delta=\delta_{1}+\delta_{2}$.

\section{A. Stability Analysis}

Let us now discuss qualitatively what happens when the mismatch $h_{\nu}$ increases. For vanishing mismatch, the system is in a fully gapped BCS state with $\Delta_{1} \equiv \Delta_{10}$ and $\Delta_{2} \equiv \Delta_{20}$, and the spin density imbalance $\delta$ is zero. With increasing $h_{\nu}$, while the BCS state is still a solution of the gap equations, there may appear another solution (Sarma) where at least one of the pairing gap $\Delta_{\nu}$ is less than the corresponding mismatch, namely $h_{\nu}>\Delta_{\nu}$. In this state, the dispersion of the quasi-particle $E_{\mathbf{k} \nu}^{\downarrow}$ becomes gapless and the system has a nonzero spin density imbalance $\delta$. Note that the normal state with vanishing condensate is always a solution of the gap equations and becomes the ground state when both $h_{1}$ and $h_{2}$ are large enough.

Different from the conventional Sarma state in singleband models, we may have two types of Sarma states in two-band systems. The first type (type I) is the solution where both mismatches are larger than the corresponding pairing gaps, namely $h_{1}>\Delta_{1}$ and $h_{2}>\Delta_{2}$. For this type, there exist gapless excitations in both bands. The second type (type II) is the solution where only one mismatch is larger than the corresponding pairing gap, $h_{1}>\Delta_{1}$ and $h_{2}<\Delta_{2}$ or $h_{1}<\Delta_{1}$ and $h_{2}>\Delta_{2}$. For this type, gapless excitations exist only in one band. We will show in the following that the stabilities of these two types of Sarma states are quite different.

A numerical example which supports the above argument is shown in Fig 1 for two symmetric bands with $U_{1}=U_{2}$. For the sake of simplicity, in our numerical examples presented here, we assume the same effective masses, chemical potentials and mismatches for the two bands, i.e., $m_{1}=m_{2} \equiv m, \mu_{1}=\mu_{2} \equiv \mu$ and $h_{1}=h_{2} \equiv h$, this means that only the total density $n$ and total spin density imbalance $\delta$ can be adjusted. We write $U_{1}$ and $U_{2}$ in terms of the s-wave scattering length $a_{\nu}$ with a momentum cutoff $k_{0}, U_{\nu}^{-1}=$ $-m /\left(4 \pi a_{\nu}\right)+\int_{|\mathbf{k}|<k_{0}} d^{3} \mathbf{k} /(2 \pi)^{3} m / \mathbf{k}^{2}$. Our qualitative conclusions do not depend on the used regularization scheme. In the case of $U_{1}=U_{2}$, the solutions of the gap equations are distributed symmetrically in the $\Delta_{1}-\Delta_{2}$ plane. Besides the familiar BCS and normal states which are, respectively, the global minimum and a local minimum in Fig 1 we have some Sarma states in the potential contour. The Sarma states $C$ and $D$ are of type I, and $C$ is the global maximum and $D$ indicates two saddle points. The type II Sarma states are marked by $A$ and $B$, corresponding, respectively, to two local minima and two saddle points.

If a solution of the gap equations is the ground state of the system, it should be the global minimum of the thermodynamic potential $\Omega^{16,26}$. The condition for a local minimum of $\Omega$ is that the matrix

$$
\mathcal{M}=\left(\begin{array}{ll}
\frac{\partial^{2} \Omega\left(\Delta_{1}, \Delta_{2}\right)}{\partial \Delta_{1}^{2}} & \frac{\partial^{2} \Omega\left(\Delta_{1}, \Delta_{2}\right)}{\partial \Delta_{1} \partial \Delta_{2}} \\
\frac{\partial^{2} \Omega\left(\Delta_{1}, \Delta_{2}\right)}{\partial \Delta_{2} \partial \Delta_{1}} & \frac{\partial^{2} \Omega\left(\Delta_{1}, \Delta_{2}\right)}{\partial \Delta_{2}^{2}}
\end{array}\right)
$$

should have only positive eigenvalues, namely $\operatorname{det} \mathcal{M}>$ 0 and $\operatorname{Tr} \mathcal{M}>0$. The second order derivatives can be evaluated as

$$
\begin{aligned}
& \frac{\partial^{2} \Omega\left(\Delta_{1}, \Delta_{2}\right)}{\partial \Delta_{\nu}^{2}}=\frac{2 J}{G} \frac{\Delta_{\bar{\nu}}}{\Delta_{\nu}}+I_{\nu}, \\
& \frac{\partial^{2} \Omega\left(\Delta_{1}, \Delta_{2}\right)}{\partial \Delta_{\nu} \partial \Delta_{\bar{\nu}}}=-\frac{2 J}{G}
\end{aligned}
$$

with the quantities $I_{\nu}$ defined as

$$
I_{\nu}=\int \frac{d^{3} \mathbf{k}}{(2 \pi)^{3}} \frac{\Delta_{\nu}^{2}}{E_{\mathbf{k} \nu}^{2}}\left[\frac{\Theta\left(E_{\mathbf{k} \nu}^{\downarrow}\right)}{E_{\mathbf{k} \nu}}-\delta\left(E_{\mathbf{k} \nu}^{\downarrow}\right)\right] .
$$

For vanishing inter-band coupling $J=0$, the stability condition becomes

$$
I_{1} I_{2}>0, \quad I_{1}+I_{2}>0 .
$$




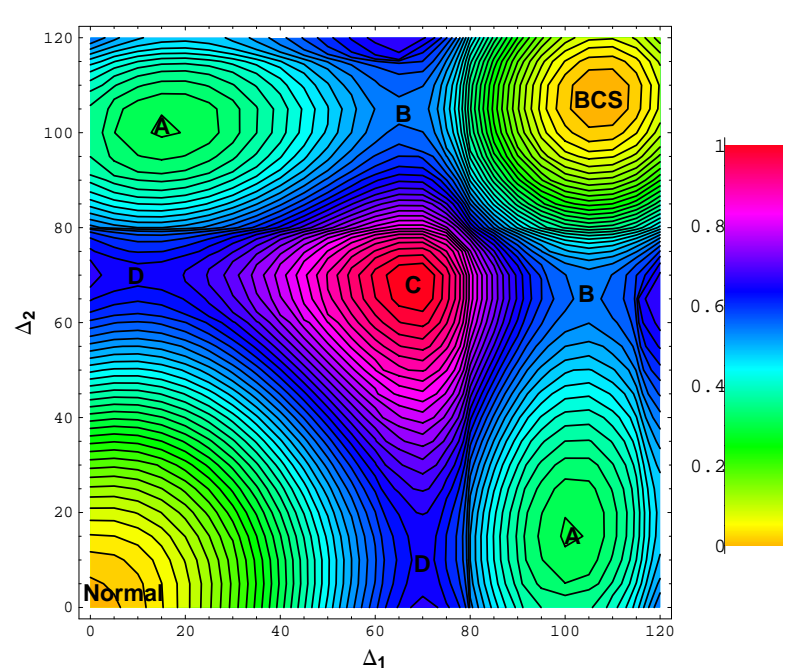

FIG. 1: The thermodynamic potential contour $\Omega\left(\Delta_{1}, \Delta_{2}\right)$ for two symmetric bands with $U_{1}=U_{2}$. A proper unit is chosen such that the Fermi energy $\epsilon_{\mathrm{F}}=200$. The values of the other parameters are $k_{0}=100 k_{\mathrm{F}}, J=10^{-4} U_{0}$ with $U_{0}=4 \pi /\left(m k_{\mathrm{F}}\right),\left(k_{\mathrm{F}} a_{1}\right)^{-1}=\left(k_{\mathrm{F}} a_{2}\right)^{-1}=-0.5$ and $h=75$, where $k_{\mathrm{F}} \simeq \sqrt{2 m \mu}$ is the Fermi momentum. The band on the right shows the relative strength of $\Omega$ corresponding to different colors. For the parameter setting, we have $U_{1}=U_{2} \simeq 0.0156 U_{0}$, and hence $J \ll U_{1}, U_{2}$.

Note that the properties of the functions $I_{1}$ and $I_{2}$ are the same as the function $I$ defined in the last section. Thus at the BCS side, namely for $a_{1}<0$ and $a_{2}<0$, the Sarma state is unstable.

Now we discuss how the inter-band coupling $J$ modifies the Sarma instability at the BCS side. For $J \neq 0$, the stability condition reads

$$
\begin{aligned}
& \frac{2 J}{G}\left(\frac{\Delta_{1}}{\Delta_{2}} I_{1}+\frac{\Delta_{2}}{\Delta_{1}} I_{2}\right)+I_{1} I_{2}>0, \\
& \frac{2 J}{G}\left(\frac{\Delta_{1}}{\Delta_{2}}+\frac{\Delta_{2}}{\Delta_{1}}\right)+I_{1}+I_{2}>0 .
\end{aligned}
$$

For the type I Sarma state with $h_{1}>\Delta_{1}$ and $h_{2}>$ $\Delta_{2}$, we have $I_{1}<0$ and $I_{2}<0$. In this case, we can exactly prove that the above two inequalities can not be satisfied simultaneously. This type of Sarma state should correspond to the maximum or saddle point of the thermodynamic potential and is hence unstable, like the points $C$ and $D$ in Fig 1 However, the situation changes for the type II Sarma state. Without loss of generality, let us discuss the case with $h_{1}>\Delta_{1}$ and $h_{2}<\Delta_{2}$. In this case, only the first band is gapless, and hence $I_{1}<0$ and $I_{2}>0$. From $I_{2}>0$, the above two inequalities are equivalent to the following one

$$
\frac{2 J}{G} \frac{\Delta_{2}}{\Delta_{1}}+I_{1}\left(1+\frac{2 J}{G I_{2}} \frac{\Delta_{1}}{\Delta_{2}}\right)>0
$$

Even though $I_{1}<0$, this condition can be satisfied, provided that a nonzero inter-band coupling $J$ is turned on. Suppose the solution of the gap equations satisfies $\Delta_{1} \ll \Delta_{2}$ and $\Delta_{1}$ is not quite close to $h_{1}$, which corresponds to the case with large polarization $\delta_{1}$, the first term in (28) is large but the modulus of the second term is relatively small, and therefore the stability condition can be satisfied, like the point $A$ at the up-left cornel in Fig[1. However, on the other hand, for $\Delta_{1} \lesssim h_{1}$ which corresponds to the case with small polarization $\delta_{1} \rightarrow 0$, the absolute value of $I_{1}$ is very large, and the Sarma state maybe unstable, which corresponds to the saddle point $B$ in the upper part of Fig:1.

We conclude that, in two-band Fermi systems with non-zero inter-band pairing interaction, the Sarma state can become at least the local minimum of the thermodynamic potential, and therefore should be a potential candidate of the ground state.

However, the condition $J \neq 0$ is not sufficient for us to have a real stable Sarma state. For the case with two symmetric bands shown in Fig.1, we found that the global minimum is always the BCS or normal state for any mismatch $h$, which means that the Sarma state can not be the ground state even though it can be a local minimum. However, this can be significantly changed if some asymmetry between the two bands, such as unequal couplings $U_{1} \neq U_{2}$, is turned on. In Fig 2 and Fig 3 we show the potential contour with $U_{1} \neq U_{2}$ for three values of $h$. In this case, the number of Sarma solutions is largely suppressed due to the asymmetry, especially the state $C$ in Fig 1 as the global maximum of $\Omega$ disappears. Without regard to the saddle points which are impossible to be stable solutions, the only Sarma state marked in Fig 2 and Fig 3 appears to be the global minimum of the system, when the mismatch $h$ is in a suitable region. From the top to the bottom in Fig, 2 and Fig 3 , when the mismatch $h$ increases, the global minimum changes from the BCS state to the Sarma state and then to the normal state. In contrast to the conventional single band model where only one first order phase transition from the BCS to normal state is predicted, we have in this two band system two first order phase transitions when $h$ increases: The first is from the BCS to Sarma state, and the second is from the Sarma to normal state. The first order phase transition from BCS to Sarma state was found in $\underline{16}$ by considering the momentum structure of the pairing gap. For ultracold atom gases, the chemical potential mismatch $h$ should be replaced by the spin population imbalance $\delta$. However, the phase structure should be essentially independent of the assembles one used $^{16.26}$, we here do not consider the case with fixed $\delta$.

Let us compare the numerical results presented in Fig 2 and Fig.3. In Fig 3, the coupling asymmetry is much larger than that in Fig 22 we have $\Delta_{10} / \Delta_{20} \simeq 1.5$ in Fig 2 and $\Delta_{10} / \Delta_{20} \simeq 4$ in Fig 3 . We find that the $h$ window for the Sarma state is wider when the coupling asymmetry becomes larger. In Fig 2, the window for Sarma state is roughly from $h=52$ to $h=71$, and the CC limit is about $h_{c} \simeq \Delta_{20}$. In Fig 3 this window is roughly from $h=20$ to $h=70$, and the CC limit is about $h_{c} \simeq 2.7 \Delta_{20}$. 

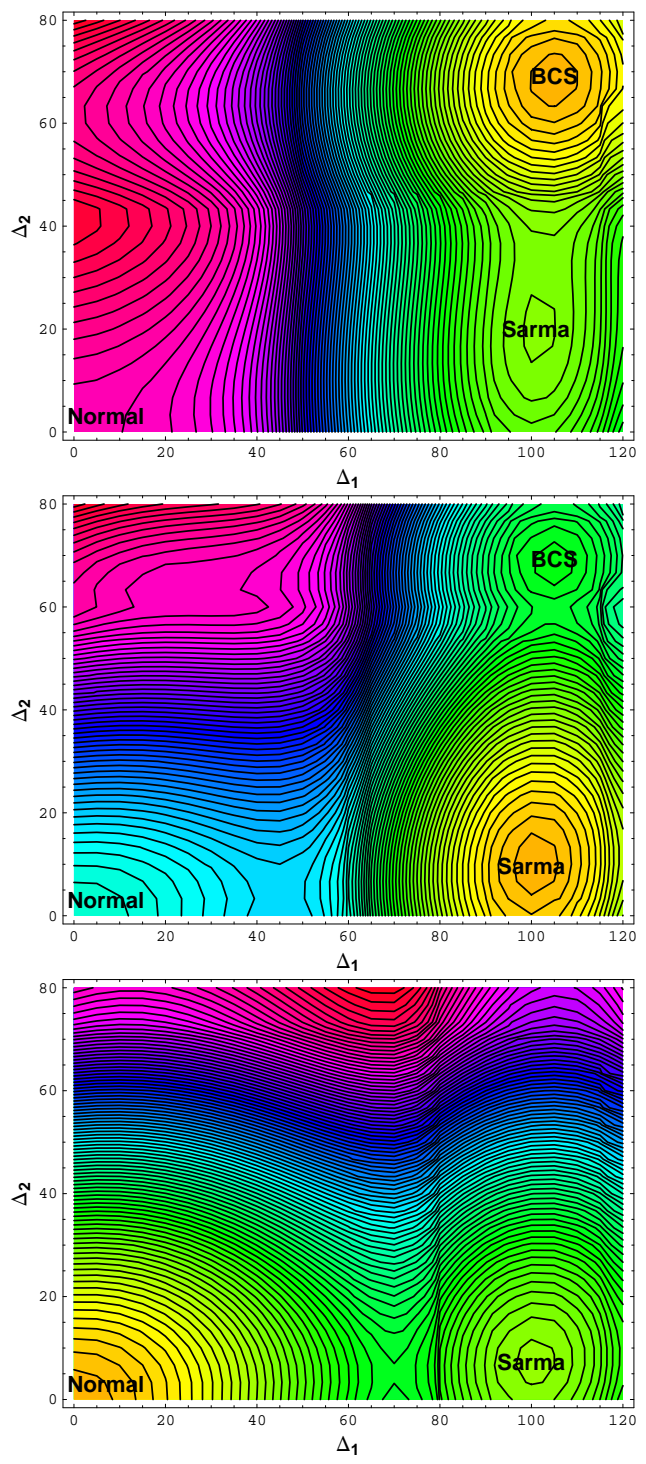

FIG. 2: The thermodynamic potential contour $\Omega\left(\Delta_{1}, \Delta_{2}\right)$ for two different bands with $\left(k_{\mathrm{F}} a_{1}\right)^{-1}=-0.5$ and $\left(k_{\mathrm{F}} a_{2}\right)^{-1}=$ -0.8 at $h=45$ (top), 60 (middle) and 75 (bottom). The other parameters are the same as that in Fig 1 .

\section{B. Solutions at Weak Coupling}

At weak coupling, the same tricks used in Section III can be employed. For convenience, we define here a function

$$
\begin{aligned}
F(\Delta, h) & =\int_{0}^{\Lambda} d \xi \frac{\Theta\left(\sqrt{\xi^{2}+\Delta^{2}}-h\right)}{\sqrt{\xi^{2}+\Delta^{2}}} \\
& \simeq \ln \frac{2 \Lambda}{\Delta}-\Theta(h-\Delta) \ln \frac{h+\sqrt{h^{2}-\Delta^{2}}}{\Delta}
\end{aligned}
$$
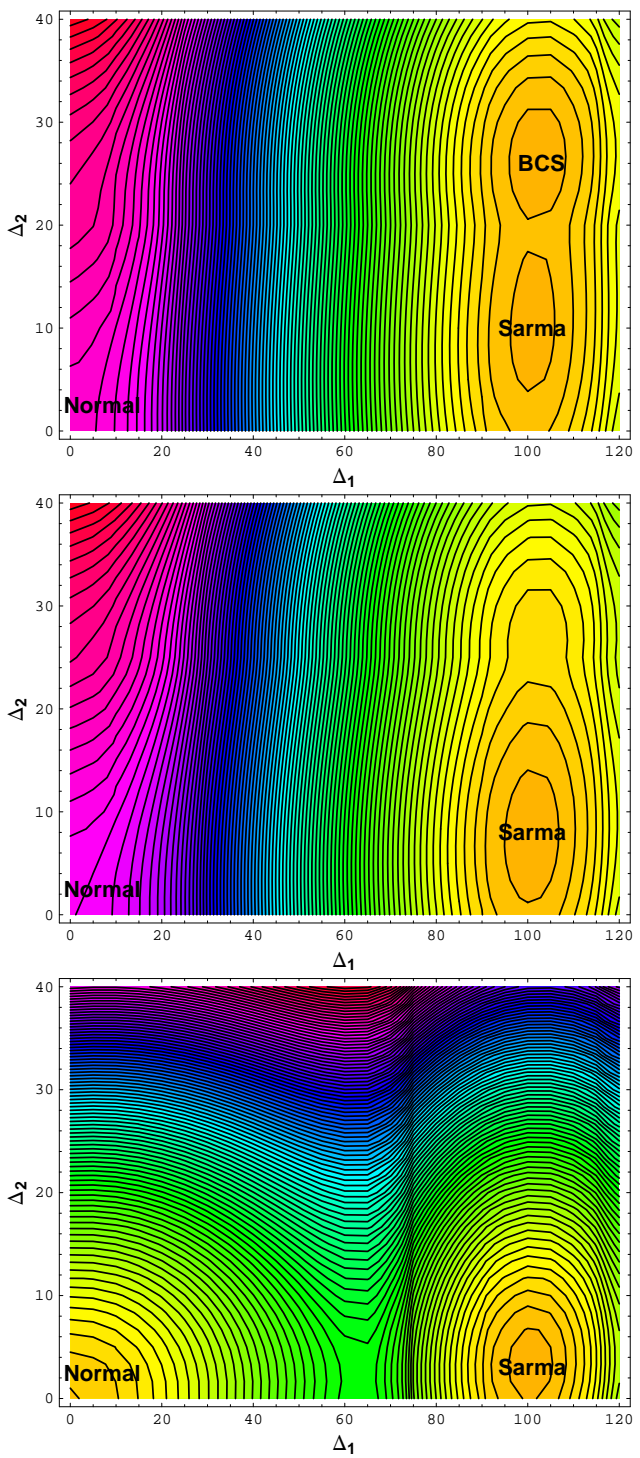

FIG. 3: The thermodynamic potential contour $\Omega\left(\Delta_{1}, \Delta_{2}\right)$ for two different bands with $\left(k_{\mathrm{F}} a_{1}\right)^{-1}=-0.5$ and $\left(k_{\mathrm{F}} a_{2}\right)^{-1}=$ -1.5 at $h=20$ (top), 25 (middle) and 70 (bottom). The other parameters are the same as that in Fig 1

and express the gap equations of our two band model in terms of it,

$$
\begin{aligned}
& {\left[\frac{U_{2}}{G N_{1}}-F\left(\Delta_{1}, h_{1}\right)\right] \Delta_{1}-\frac{J}{G N_{1}} \Delta_{2}=0,} \\
& {\left[\frac{U_{1}}{G N_{2}}-F\left(\Delta_{2}, h_{2}\right)\right] \Delta_{2}-\frac{J}{G N_{2}} \Delta_{1}=0,}
\end{aligned}
$$

where $N_{1}$ and $N_{2}$ are the densities of state at the Fermi surfaces for the two bands. Unlike the single band model, the above coupled gap equations can not be solved analytically. With the numerical solutions $\Delta_{1}$ and $\Delta_{2}$, the 
thermodynamic potential can be evaluated as

$$
\Omega=-\sum_{\nu}\left[\frac{N_{\nu}}{2} \Delta_{\nu}^{2}+\Theta\left(h_{\nu}-\Delta_{\nu}\right) N_{\nu} h_{\nu} \sqrt{h_{\nu}^{2}-\Delta_{\nu}^{2}}\right]
$$

For the sake of simplicity, we consider the case $h_{1}=$ $h_{2}=h$ which corresponds to the realistic two-band superconductors in strong magnetic field. Let us assume $U_{1} N_{1}>U_{2} N_{2}$ which leads to $\Delta_{1}>\Delta_{2}$. According to the stability analysis, we have three possible ground states: 1)The normal state with $\Delta_{1}=\Delta_{2}=0 ; 2$ )The gapped BCS state with energy gaps $\Delta_{1} \equiv \Delta_{10}>h$ and $\Delta_{2} \equiv \Delta_{20}>h$; 3) The gapless Sarma state where only $\Delta_{1}>h$ but $\Delta_{2}<h$. We focus here on the case with $\Delta_{2} \ll \Delta_{1}$ and $J \ll \sqrt{U_{1} U_{2}}$. In this case, the solution of $\Delta_{1}$ is approximately independent of $h$ and is given by

$$
\Delta_{1}=\Delta_{10} \simeq 2 \Lambda e^{-1 /\left(U_{1} N_{1}\right)},
$$

and the Sarma solution for $\Delta_{2}$ is determined by the following equation

$$
\ln \frac{\Delta_{20}}{h+\sqrt{h^{2}-\Delta_{2}^{2}}}=\frac{J \Delta_{10}}{U_{1} U_{2} N_{2}}\left(\frac{1}{\Delta_{20}}-\frac{1}{\Delta_{2}}\right)
$$

where $\Delta_{20}$ is obtained by the equation

$$
\frac{1}{U_{2} N_{2}}-\ln \frac{2 \Lambda}{\Delta_{20}}=\frac{J}{U_{1} U_{2} N_{2}} \frac{\Delta_{10}}{\Delta_{20}} .
$$

We have numerically checked that the above approximation is sufficiently good for the scaled solution $y=$ $\Delta_{2} / \Delta_{20}$ as a function of $x=h / \Delta_{20}$. Note that for $J=0$ the conventional Sarma solution $y=\sqrt{2 x-1}(0.5<x<$ 1 ) is recovered, but for $J \neq 0$ the Sarma solution is qualitatively changed: $y=0$ can not be a solution and there exist solutions for $x>1$. The solutions for both cases of $J=0$ and $J \neq 0$ are illustrated in Fig鸟. We find that for $J \neq 0$ the Sarma solution is quite different from the conventional result. Unlike the well-known Sarma solution which exists in the region $0.5<x<1$, for $J \neq 0$ the Sarma state exists almost in the region $x>1$ where the BCS solution $y=1$ disappears. Obviously, in a narrow region $x \lesssim 1$ there exists a branch of the conventional type which is unstable, and the multi-value behavior of $y$ means a first order BCS-Sarma phase transition at a critical field $x_{1}$ which is slightly smaller than 1 .

To discuss the thermodynamic stability of the Sarma state, we then need to compare it with the normal state. In the case of $\Delta_{2}<h$, we find

$$
\begin{aligned}
\Omega_{\mathrm{S}}-\Omega_{\mathrm{N}} & =\frac{N_{1}}{2}\left(2 h^{2}-\Delta_{1}^{2}\right) \\
& +\frac{N_{2}}{2}\left(2 h^{2}-\Delta_{2}^{2}-2 h \sqrt{h^{2}-\Delta_{2}^{2}}\right) .
\end{aligned}
$$

Some analytical estimations can be made. For large asymmetry $\Delta_{2} \ll \Delta_{1}$, around the $h$-window $h \sim \Delta_{20}$ but $h \ll \Delta_{10}$ for the Sarma state, the sign of the quantity $\Omega_{\mathrm{S}}-\Omega_{\mathrm{N}}$ is dominated by the first term, if $N_{1}$ is not

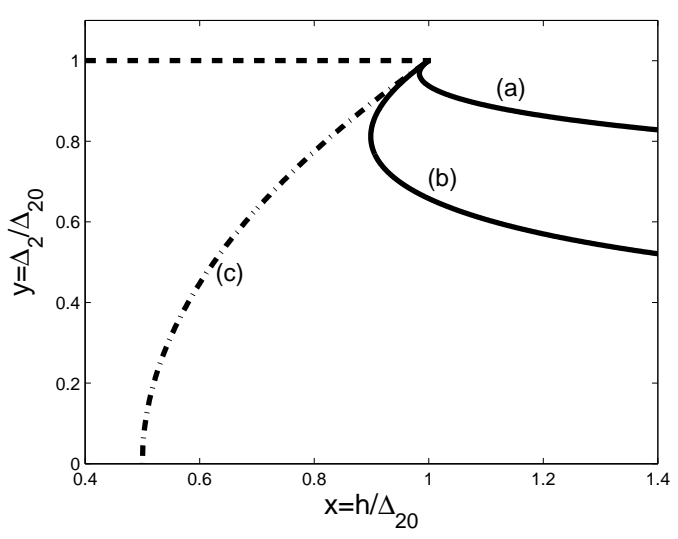

FIG. 4: The solution $y=\Delta_{2} / \Delta_{20}$ to the gap equations as a function of $x=h / \Delta_{20}$. The dashed line denotes the BCS solution $y=1$ in the region $0<x<1$. The solid lines (a) and (b) are the Sarma solutions for $J \neq 0$. In the calculations, we take $N_{1} / N_{2}=1.5$ and $J / \sqrt{U_{1} U_{2}}=0.07$. For (a) we take $\left(U_{1} N_{1}\right) /\left(U_{2} N_{2}\right)=3$ which leads to $\Delta_{10} / \Delta_{20} \simeq 9$, and for (b) we have $\left(U_{1} N_{1}\right) /\left(U_{2} N_{2}\right)=1.67$ and hence $\Delta_{10} / \Delta_{20} \simeq 3$. The dot-dashed line (c) is the conventional Sarma solution for $J=0, y=\sqrt{2 x-1}$ in the region $0.5<x<1$.

much smaller than $N_{2}$. In this case, the BCS solution is absent and the Sarma state is the stable ground state. This argument confirms our conclusion from the numerical results in Fig 2 and Fig 33. The $h$ window for stable Sarma state is wider when the asymmetry between the two bands becomes larger. This means that the CC limit of such a two-band superconductor can be much higher than the conventional value $h_{c}=0.707 \Delta_{20}$.

\section{SUMMARY}

We have studied the stability of Sarma state in twoband Fermi systems via both the stability analysis and analytical solution at weak coupling. From the stability analysis, the Sarma state can be the minimum of the thermodynamic potential and hence a possible candidate of the ground state, if the inter-band exchange interaction is turned on. Both numerical and analytical studies show that, a large asymmetry between the two bands or the two pairing gaps is an important condition for thermodynamic stability of the Sarma state. When the condition is satisfied, two first order phase transitions will occur when the mismatch increases, one is from the BCS to Sarma state at a lower mismatch and the other is from the Sarma to normal state at a higher mismatch. Our predictions could be tested in multi-band superconductors and ultracold atom gases, and such a gapless superconductor may have many unusual properties, such as magnetism and large spin susceptibility 27,28 .

Acknowledgments: We thank W.V.Liu and M.Iskin for useful communications and Y.Liu and X.Hao for the 
help in numerical calculations. The work is supported by the NSFC Grants 10575058 and 10735040 and the
National Research Program Grant 2006CB921404.
1 G.Sarma, J.Phys.Chem.Solid 24,1029(1963)

2 P.Fulde and R.A.Ferrell, Phys. Rev. A135, 550(1964); A.I.Larkin and Yu.N.Ovchinnikov, Sov.Phys. JETP 20, 762(1965)

3 B.S.Chandrasekhar, Appl. Phys. Lett. 1,7(1962); A.M.Clogston, Phys. Rev. Lett. 9, 266(1962)

${ }^{4}$ K. Gloos, R.Modler, H.Schimanski, C.D.Bredl, C.Geibel, F.Steglich, A.I.Buzdin, N.Sato and T.Komatsubara, Phys. Rev. Lett. 70, 501 (1993); R. Modler, P.Gegenwart, M.Lang, M.Deppe, M.Weiden, T. Lhmann, C.Geibel, F.Steglich, C.Paulsen, J.L.Tholence, N.Sato, T.Komatsubara, Y.nuki, M.Tachiki and S.Takahashi, Phys. Rev. Lett. 76, 1292(1996); A.Bianchi, R.Movshovich, C.Capan, P. G.Pagliuso and J.L.Sarrao, Phys. Rev. Lett. 91 (2003) 187004

${ }^{5}$ J. L. O'Brien, H. Nakagawa, A. S. Dzurak, R. G. Clark, B. E. Kane, N. E. Lumpkin, R. P. Starrett, N. Muira, E. E. Mitchell, J. D. Goettee, D. G. Rickel, and J. S. Brooks, Phys. Rev. B 61, 1584 (2000)

${ }^{6}$ L. Balicas, J.S.Brooks, K.Storr, S.Uji, M.Tokumoto, H.Tanaka, H. Kobayashi, A.Kobayashi, V.Barzykin and L.P.Gor'kov, Phys. Rev. Lett. 87, 067002(2001); M. A. Tanatar, T. Ishiguro, H. Tanaka and H. Kobayashi, Phys. Rev. B 66, 134503 (2002)

7 M.W.Zwierlein, A.Schirotzek, C.H. Schunck and W. Ketterle, Science 311, 492(2006); G.B.Partridge, W.Li, R.I.Kamar, Y.-an Liao, R.G. Hulet , ibid.311, 503(2006)

8 P.F.Bedaque, H. Caldas and G. Rupak, Phys. Rev. Lett. 91, 247002(2003); A.Sedrakian, J. Mur-Petit, A. Polls and H. Müther, Phys. Rev. A72, 013613(2005); L.He, M.Jin and P.Zhuang, Phys. Rev. B73, 214527(2006); Phys.Rev.B74, 214516(2006)

9 C.H.Pao, S.-T. Wu and S.-K.Yip, Phys. Rev. B73, 132506(2006); D.E.Sheehy and L.Radzihovsky, Phys. Rev. Lett. 96, 060401(2006); D.T.Son and M.A.Stephanov, Phys. Rev. A74, 013614(2006); H.Hu and X.Liu, ibid.73, 051603(R)(2006); M.Mannarelli, G. Nardulli and M. Ruggieri, ibid.74, 033606(2006); A.Bulgac and M.M.Forbes, ibid.75, 031605(R)(2007);

10 J.Kinnunen, L.M.Jensen and P.Törmä, Phys. Rev. Lett. 96, 110403(2006); P.Pieri and G.C.Strinati, ibid.96, 150404(2006); T.Silva and E.Mueller, ibid.97, 070402(2006); T.Mizhushima, T.Mizushima and M. Ichioka, ibid.97, 120407(2006)

11 D.E.Sheehy and L.Radzihovsky, Ann. Phys. (N.Y.)322,
$1790(2007)$

12 A.J.Leggett, in Modern trends in the theory of condensed matter, Springer-Verlag, Berlin, 1980, pp.13-27

13 M.Huang, P.Zhuang, and W.Chao, Phys. Rev. D67, 065015(2003); I.Shovkovy and M.Huang, Phys. Lett.B564, 205(2003); M.Alford, C.Kouvaris and K. Rajagopal, Phys. Rev. Lett. 92, 222001(2004)

14 D.Ebert and K.G.Klimenko, J.Phys.G32,599(2006); L.He, M.Jin and P.Zhuang, Phys.Rev.D74, 036005(2006).

15 W.V.Liu and F.Wilczek, Phys. Rev. Lett.90, 047002(2003)

16 M.M.Forbes, E.Gubankova, W.V.Liu and F.Wilczek, Phys. Rev. Lett.94, 017001(2005)

17 E.Taylor, A.Griffin and Y.Ohashi, Phys. Rev. A76, 023614 (2007)

18 H. Suhl, B.T.Matthias and L.R.Walker, Phys. Rev. Lett.3, $552(1959)$

19 V.M.Loktev, R. M. Quick and S. G. Sharapov, Phys. Rept. 349,1(2001)

20 M. Iavarone, G. Karapetrov, A. E. Koshelev, W. K. Kwok, G. W. Crabtree, D. G. Hinks, W. N. Kang, E.-M Choi, H. J. Kim, H.-J. Kim, and S. I. Lee, Phys. Rev. Lett.89, 187002 (2001); F. Bouquet, Y. Wang, I. Sheikin, T. Plackowski, A. Junod, S. Lee and S. Tajima, ibid.89, 257001 (2002); S.Tsuda, T. Yokoya, Y. Takano, H. Kito, A. Matsushita, F. Yin, J. Itoh, H. Harima and S. Shin, ibid.91, 127001 (2003); J. Geerk, R. Schneider, G. Linker, A. G. Zaitsev, R. Heid, K.-P. Bohnen and H. v. Loehneysen, ibid.94, 227005 (2005)

21 M. Köhl, H. Moritz, T. Stöferle, K. Günter, and T. Esslinger, Phys. Rev. Lett.94, 080403(2005); L.M.Duan, Phys. Rev. Lett.95, 243202 (2005)

${ }^{22}$ M. Iskin and C. A. R. Sá de Melo, Phys. Rev.B72, 024512 (2005); ibid.74, 144517(2006)

23 J.-P. Martikainen, E. Lundh and T. Paananen, Phys. Rev.A78, 023607(2008)

24 A.I.Akhiezer, A.A.Isayev, S.V.Peletminsky and A.A. Yatsenko, Phys. Lett. B451, 430(1999)

25 R.Casalbuoni and G.Nardulli, Rev.Mod.Phys.76, 263(2004)

26 D.E.Sheehy and L.Radzihovsky, Phys. Rev. B75, 136501(2007)

27 S.Takada and T.Izuyama, Prog.Theor.Phys.41,635(1969).

28 L.He, M.Jin and P.Zhuang, Phys.Rev.B73, 024511(2006) 